\title{
Role of De Novo DNA Methyltransferases in Initiation of Genomic Imprinting and X-Chromosome Inactivation
}

\author{
M. Kaneda, ${ }^{* \dagger}$ T. SAdo, ${ }^{* \dagger}$ K. Hata, ${ }^{* \dagger}$ M. OKano, ${ }^{\S} \|$ N. Tsujimoto, ${ }^{\| \#}$ \\ E. LI, "\# AND H. SASAKI ${ }^{* \dagger}$ \\ *Division of Human Genetics, Department of Integrated Genetics, National Institute of Genetics, Research \\ Organization of Information and Systems (ROIS), Mishima 411-8540, Japan; 'Department of Genetics, School of \\ Life Science, Graduate University for Advanced Studies (SOKENDAI), Mishima 411-8540, Japan; ${ }^{\ddagger}$ RESTO, \\ Japan Science and Technology Agency (JST), Kawaguchi 332-0012, Japan; ${ }^{\S}$ Laboratory for Mammalian \\ Epigenetic Studies, Center for Developmental Biology, RIKEN, Kobe 650-0047, Japan; "Cardiovascular Research \\ Center, Massachusetts General Hospital, Harvard Medical School, Charlestown, Massachusetts 02129
}

DNA methylation is an epigenetic mechanism that plays a key role in regulation of developmental gene expression, maintenance of genomic integrity, genomic imprinting, and $\mathrm{X}$-chromosome inactivation (X-inactivation) in mammals. Methylation of mammalian genomic DNA occurs almost exclusively at the cytosine of $\mathrm{CpG}$ dinucleotides. The $\mathrm{CpG}$ methylation pattern of the mammalian genome is created and maintained by a combination of de novo DNA methyltransferases, Dnmt $3 a$ and Dnmt $3 b$, and a maintenance DNA methyltransferase Dnmt1. Targeted disruption of these DNA methyltransferase genes in mice results in embryonic or early postnatal lethality, indicating that they are essential for normal mammalian development (Li et al. 1992; Okano et al. 1999).

Genomic imprinting and X-inactivation are the wellcharacterized, major epigenetic phenomena of mammals that regulate allelic expression of autosomal genes and Xlinked genes, respectively (Lyon 1961; Reik and Walter 2001). Both phenomena are known to be crucial for normal mammalian development. Imprinting is initiated during male and female gametogenesis, marking a subset of autosomal genes (up to a few hundred) in a sex-specific way (paternal and maternal imprinting). The imprinted genes show either paternal-specific or maternal-specific monoallelic expression in the offspring (Reik and Walter 2001). Thus imprinting is dependent on the sex of the parent from which the gene is derived, but not on the sex of the individual that carries the gene. By contrast, X-inactivation is a dosage compensation mechanism found only in females, which equalizes the X-linked gene dosage between males (with one $\mathrm{X}$ and one $\mathrm{Y}$ chromosome) and females (with two $X$ chromosomes) (Lyon 1961). In the embryo proper (the epiblast lineages), $\mathrm{X}$-inactivation is initiated during early development, leading to random inactivation of either the paternal or the maternal $\mathrm{X}$ chromosome. However, in the extraembryonic lineages (trophoblast and primitive endoderm derivatives) of mice, preferential inactivation of the paternal $\mathrm{X}$ chromosome occurs (Takagi and Sasaki 1975). Thus X-inactivation

\footnotetext{
\#Present address: Novartis Institute for Biomedical Research, Cambridge, Massachusetts 02139 .
}

can be subject to genomic imprinting (imprinted $\mathrm{X}$-inactivation). Like the imprinting of autosomes, the imprinting of X chromosome is thought to occur in the parental germ line.

Previous studies with the mouse embryos and ES cells deficient for Dnmt1 showed that DNA methylation plays an essential role in the maintenance of genomic imprinting and $\mathrm{X}$-inactivation in the embryo proper (Table 1) $(\mathrm{Li}$ et al. 1993; Beard et al. 1995; Panning and Jaenisch, 1996; Sado et al. 2000). By contrast, in the trophoblast, the role of DNA methylation seems more relaxed (Table 1) (Caspery et al. 1998; Tanaka et al. 1999; Sado et al. 2000). However, whether DNA methylation is involved in their initiation has not been addressed. If DNA methylation were to play a role in the initiation step, Dnmt $3 a$ or Dnmt3b (or both) should be the key players because these are the enzymes that establish new genomic methylation patterns (Okano et al. 1999). We therefore asked whether Dnmt $3 a$ and/or Dnmt $3 b$ is involved in the initiation of autosomal imprinting and $\mathrm{X}$-inactivation using the cells and embryos deficient for these genes. The Cre-loxP conditional gene knockout system was particularly useful because of the early lethality of conventional Dnmt3a or Dnmt3b knockout mice (Okano et al. 1999). In this article, we summarize the results obtained from these experiments and discuss the role of de novo DNA methylation in the initiation of the two epigenetic phenomena.

\section{ROLE FOR DNA METHYLATION IN IMPRINTING OF AUTOSOMAL GENES IN THE PARENTAL GERM LINE}

Several lines of evidence suggest a role for DNA methylation in the initiation of autosomal imprinting. Mice deficient for Dnmt3L, a protein sharing homology with Dnmt3a and Dnmt3b but lacking methyltransferase activity, showed a failure in establishment of oocyte-specific methylation imprints (Bourc'his et al. 2001; Hata et al. 2002). Offspring from such females showed loss of monoallelic expression of the maternally imprinted genes and died around embryonic day 10.5 (E10.5). Since Dnmt3L protein can interact with Dnmt3a and Dnmt3b enzymes in transfected cells (Hata et al. 2002), it has been 
KANEDA ET AL.

Table 1. DNA Methylation in Autosomal Imprinting and X-Chromosome Inactivation

\begin{tabular}{|c|c|c|c|}
\hline Phenomenon & Step & Tissue & $\begin{array}{l}\text { DNA methylation } \\
\text { required? }\end{array}$ \\
\hline \multirow[t]{3}{*}{ Autosomal imprinting } & Initiation & Germ line & Yes \\
\hline & Maintenance & Embryo proper & Yes \\
\hline & Maintenance & Extraembryonic & $\mathrm{Yes} / \mathrm{No}^{\mathrm{a}}$ \\
\hline \multirow[t]{2}{*}{ Random X-inactivation } & Initiation & Epiblast & $\underline{\text { No }}$ \\
\hline & Maintenance & Embryo proper & Yes \\
\hline \multirow{2}{*}{ Imprinted $\mathrm{X}$-inactivation } & Initiation & Germ line & No?/? ${ }^{\mathrm{b}}$ \\
\hline & Maintenance & Extraembryonic & No \\
\hline
\end{tabular}

proposed that Dnmt3L may regulate maternal imprinting via the de novo methyltransferases. Consistent with this model, a [Dnmt $3 a^{-/-}$, Dnmt $\left.3 b^{+/-}\right]$ovary transplanted into a wild-type female failed to establish the oocyte-specific (maternal) methylation imprints (Hata et al. 2002).

We wanted to investigate the role of Dnmt3a and/or $D n m t 3 b$ in the initiation step of imprinting in more detail by disrupting the genes in male and female germ cells. A big problem was that the conventional Dnmt $3 b$ knockout mice are embryonic lethal and the conventional Dnmt3a knockout mice die around 3-4 weeks of age, before reaching the reproductive stage (Okano et al. 1999). We therefore could not examine the gametes of these mice or the offspring derived from them. To overcome this problem, we took advantage of the Cre-loxP conditional knockout technology and disrupted the Dnmt 3 genes in a germ-cell-specific way, leaving the genes intact in most somatic cells (Fig. 1). We used the tissue nonspecific alkaline phosphatase (TNAP)-Cre knockin mice, which express the Cre recombinase in germ cells from E9.5 to late gestation (Lomeli et al. 2000). Although expression of TNAP-Cre was not strictly germ-cell-specific, we could

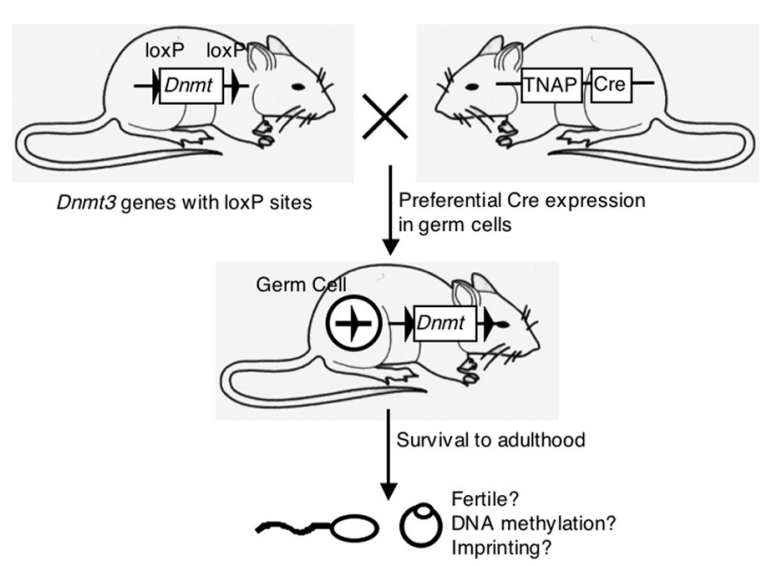

Figure 1. Conditional knockout of the de novo DNA methyltransferase genes Dnmt $3 a$ and Dnmt $3 b$ in mouse germ cells. The conditional mice with two loxP sites were produced by gene targeting and crossed with TNAP-Cre mice, which express the Cre recombinase in germ cells. The resulting conditional knockout mice survived to adulthood, and thus we could examine their germ cells (in both male and female mutants) and offspring (derived from the female mutants). For details, see Kaneda et al. (2004). derive conditional knockout mice ([Dnmt $3 a^{2 l o x / 1 l o x}$, TNAP-Cre] and [Dnmt $3 b^{2 l o x / 1 l o x}$, TNAP-Cre], where 2lox represents the functional allele and 1lox represents the nonfunctional allele) that can survive to adulthood (Fig. 1) (Kaneda et al. 2004).

When the [Dnmt3a 2 lox/1lox , TNAP-Cre] females were crossed with wild-type males, no live pups were obtained. Subsequent studies revealed that all embryos died around E10.5 with various developmental defects, such as open neural tube, lack of branchial arches, and impediment of blood circulation (Kaneda et al. 2004). We examined the methylation status of the differentially methylated regions (DMRs) of the imprinted genes in the E10.5 embryos. The DMRs normally methylated on the maternal allele, such as those of Snrpn, Igf2r, and Pegl, were found to be unmethylated (Kaneda et al. 2004). By contrast, the methylation status of the paternally methylated H19 and Rasgrf1 DMRs was unaffected. We then examined the expression of the maternally imprinted genes in the same embryos and found that $p 57^{k i p 2}(C d k n l c)$ and $\operatorname{Ig} f 2 r$ are silenced, consistent with a loss of expression from the normally active maternal alleles (Kaneda et al. 2004). We also found that expression of Peg1, Snrpn, and $P e g 3$ is increased, with a derepression of the normally silent maternal alleles (Kaneda et al. 2004). Thus, the Dnmt $3 a$ conditional mutant females fail to establish the oocyte-specific imprints at the maternally imprinted loci.

The [Dnmt3a $a^{2 l o x} / 1 l o x$, TNAP-Cre] males showed impaired spermatogenesis (Kaneda et al. 2004). Histological examinations showed that the testes from the mutant males contain a slightly reduced number of spermatogonia in the seminiferous tubules at postnatal day 11 (P11) (Kaneda et al. 2004). However, at 11 weeks of age, they contained only few spermatogonia and no spermatocytes, spermatids, or spermatozoa (Kaneda et al. 2004). Because of the azoospermia, we could not obtain offspring to examine. We therefore used the laser-microdissection technology to collect spermatogonia from histological sections of the P11 testes and analyzed three paternally methylated DMRs by bisulfite sequencing. We found that the spermatogonia from the Dnmt $3 a$ conditional mutant males lacked methylation at the normally methylated H19 DMR and Dlk1-Gtl2 intergenic DMR (Kaneda et al. 2004). The normally methylated DMR of Rasgrf1 was slightly less methylated than in wild-type spermatogonia (Kaneda et al. 2004). These results indicate that Dnmt3a 
is required for the initiation of imprinting in both paternal and maternal germ lines.

We also generated [Dnmt3 $b^{2 l o x} / 1$ lox, TNAP-Cre $]$ mice, which were found to be phenotypically normal. When the [Dnmt $3 b^{2 l o x / 1 l o x}$, TNAP-Cre] males and females were crossed with wild-type partners, healthy pups were obtained (Kaneda et al. 2004). We analyzed the DMRs for the allelic methylation difference in these pups but all was found to be normal (Kaneda et al. 2004). Thus, so far there is no evidence that $D n m t 3 b$ is involved in the initiation of imprinting in the parental germ line.

\section{ROLE FOR DNA METHYLATION IN IMPRINTING OF Mash2 (Ascl2) IN THE MATERNAL GERM LINE}

The mouse Mash2 (Ascl2) gene encodes a transcription factor of the basic helix-loop-helix class that is essential for extraembryonic development (Guillemot et al. 1994). Mash2 is located within a large imprinted cluster on mouse distal chromosome 7 and is exclusively expressed from the maternal allele (Guillemot et al. 1995). However, the imprinting of this gene is unique in that its maintenance is highly resistant to hypomethylation, as shown by the analysis of the Dnmt1-deficient embryos (Caspery et al. 1998; Tanaka et al. 1999). It is therefore interesting to ask whether the imprinting of this gene is initiated normally in the germ line of [Dnmt $3 a^{2 l o x / 1 l o x}$, TNAP-Cre] females. (It is known that Mash2 is a maternally imprinted gene.) Expression of Mash2 and $p 57^{k i p 2}(C d k n 1 c)$, as well as Gapd (a nonimprinted control), was examined in the trophoblast of E9.5 embryos obtained from the [Dnmt $3 a^{2 l o x / 1 l o x}$, TNAP-Cre] females crossed with wild-type males. The results showed that both Mash2 and p5 $7^{\text {kip } 2}$ are silenced in the trophoblast whereas Gapd expression is unaffected (Fig. 2), indicating that the initiation of Mash2 imprinting in the maternal germ line does require Dnmt3a. This finding is consistent with the fact that the imprinting of Mash2 is controlled by an imprinting center located in an intron of Kvlqt1 (Kcnq1) (Fitzpatrick et al. 2002), which is methylated in oocytes but not in sperm.

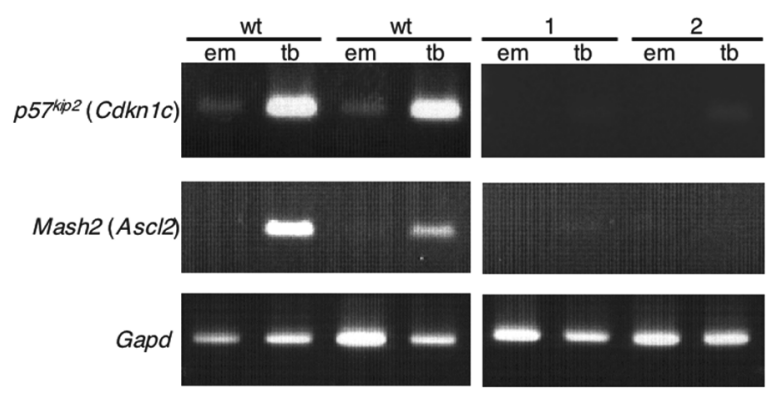

Figure 2. A maternally imprinted gene Mash2 is silenced in the trophoblast of embryos derived from [Dnmt $3 a^{2 \text { lox } / 1 l o x}$, TNAPCre] mothers. Expression of Mash2, as well as $p 57^{k i p 2}$, was examined by RT-PCR in the embryo proper (em) and trophoblast (tb) of two conceptuses $(1,2)$. The data suggest that the initiation of Mash2 imprinting in the maternal germ line requires DNA methylation. Gapd is a nonimprinted housekeeping control. wt, wild-type.

\section{ROLE FOR DNA METHYLATION IN INITIATION OF RANDOM X-CHROMOSOME INACTIVATION}

We next asked whether the initiation of random X-inactivation in the embryo proper (epiblast lineages) and ES cells requires the de novo DNA methyltransferases. In the initiation step of X-inactivation, a noncoding RNA, X-inactive specific transcript (Xist), is upregulated on the future inactive $\mathrm{X}$ chromosome. The Xist RNAs then coat the entire chromosome and presumably recruit factors required for heterochromatin formation (Brockdorff 2002). By contrast, Xist is stably silenced on the active X chromosome. Although the maintenance of the silenced state of Xist requires Dnmt1, monoallelic Xist expression and subsequent $X$-inactivation can occur normally in ES cells deficient for Dnmt1 (Beard et al. 1995; Panning and Jaenisch 1996). We therefore examined whether X-inactivation can occur normally in the epiblast lineages of [Dnmt $3 a^{-/-}$, Dnmt $\left.3 b^{-/-}\right]$embryos and in differentiating $\left[\right.$ Dnmt3a ${ }^{-/-}$, Dnmt3b $\left.b^{-/}\right]$ES cells (Sado et al. 2004).

We found by RNA fluorescence in situ hybridization (FISH) that monoallelic Xist expression is appropriately initiated in most cells of the $\left[\right.$ Dnmt $3 a^{-/-}$, Dnmt $\left.3 b^{-/}\right]$female embryos at E9.5 (Sado et al. 2004). Furthermore, a cytological analysis showed that one of the two X chromosomes unanimously replicates late in $\mathrm{S}$ phase. In addition, one X chromosome was hypoacetylated at histone $\mathrm{H} 4$ in the mutant female embryos as in wild-type female embryos, as revealed by immunostaining. These results indicate that random $\mathrm{X}$-inactivation occurred appropriately in the absence of the de novo methyltransferases (Sado et al. 2004). The Xist promoter was extensively hypomethylated in these embryos.

In the above experiments, however, we observed ectopic accumulation of Xist RNA (on the single X chromosome in males and on both $\mathrm{X}$ chromosomes in females) in a subset of cells (typically 4-5\%) (Sado et al. 2004). Do these ectopic signals arise from inappropriate activation of Xist at the onset of X-inactivation or from derepression of a once silenced Xist locus? To address this question, we examined Xist RNA accumulation in the course of differentiation of $\left[\right.$ Dnmt $3 a^{-1-}$, Dnmt $\left.3 b^{-/-}\right]$male ES cells (Sado et al. 2004). Although the single $X$ chromosome was never coated with Xist RNA in undifferentiated state, ectopic Xist accumulation was detected in $3.2 \%$ and $16.8 \%$ of cells at day 2 and day 5 of differentiation, respectively. At day 12 of differentiation, a surprisingly high percentage (68\%) of cells from $\left[\right.$ Dnmt $3 a^{-1-}$, Dnmt $3 b^{-/-}$] embryoid bodies showed ectopic accumulation, suggesting a progressive derepression of the unmethylated Xist locus. These observations establish that de novo DNA methylation is not required for the initial silencing of Xist but is necessary for stabilizing the silenced state of Xist (Sado et al. 2004).

\section{ROLE FOR DNA METHYLATION IN INITIATION OF IMPRINTED X-CHROMOSOME INACTIVATION}

In contrast to the random $\mathrm{X}$-inactivation in the embryo proper, an imprinted X-inactivation occurs in the ex- 
traembryonic lineages (Takagi and Sasaki 1975). This seems to be due to an imprint on the maternal X chromosome $(\mathrm{Xm})$ to remain active, as well as an imprint on the paternal $\mathrm{X}$ chromosome (Xp) to inactivate. The paternal imprint on the Xp can be reversed: XpO mice are developmentally retarded but viable and fertile (which indicates that the $\mathrm{Xp}$ is active) (Thornhill and Burgoyne 1993) and androgenetic embryos with two Xp chromosomes can undergo random $\mathrm{X}$-inactivation in the extraembryonic tissues (Okamoto et al. 2000). The maternal imprint on the Xm may be more rigid: Genetic experiments using Robertsonian translocations showed that, in embryos carrying two Xm chromosomes, both remain active in the extraembryonic tissues and that such embryos die early because of poor development of the extraembryonic tissues (Goto and Takagi 1998). Furthermore, nuclear transplantation experiments showed that the maternal imprint is set on the Xm during oocyte growth (Tada et al. 2000), just as the maternal imprints on autosomes. Recently, it was found that imprinted X-inactivation is observed from the two-cell or four-cell stage (Huynh and Lee 2003; Okamoto et al. 2004) and it is proposed that the preinactivated state of the $\mathrm{Xp}$ is carried over from the paternal germ line (Huynh and Lee 2003).

We wanted to ask whether the de novo DNA methyltransferases plays a role in imprinting $\mathrm{X}$ chromosome in the male and female germ lines. Since disruption of Dnmt3a in the male germ line results in azoospermia (Kaneda et al. 2004), we could ask only whether its disruption in the female germ line has an effect on imprinted $\mathrm{X}$-inactivation. As described above, embryos conceived by the [Dnmt $3 a^{2 l o x / 1 l o x}$, TNAP-Cre] females survived until E10.5 (Kaneda et al. 2004), indicating that the Xlinked gene dosage was appropriately controlled, or nearly so, during early development. We recently obtained several embryos from [Dnmt $3 a^{2 l o x / 1 l o x}$, Dnmt $3 b^{2 l o x / 1 l o x}$, TNAP-Cre] females and found that their phenotype is almost identical with that of the above embryos. Two X-inactivation patterns are envisaged in these embryos. One is the normal imprinted $\mathrm{X}$-inactivation, which suggests that the loss of the de novo DNA methyltransferases in the female germ line had no effect on the $\mathrm{Xm}$. The other is a random X-inactivation, which suggests that the Xm had lost the imprint and then the counting and choice mechanisms were switched on. The previous findings that the $\mathrm{Xm}$ derived from nongrowing oocytes resembled the normal Xp (Tada et al. 2000) and the presence of two Xp chromosomes resulted in random $\mathrm{X}$-inactivation (Okamoto et al. 2000) appear to support the latter idea.

To distinguish between the two possibilities, we made use of mice carrying an X-linked green fluorescent protein (GFP) transgene ( $\mathrm{X}^{\mathrm{GFP}}$ ) (Takagi et al. 2002). [Dnmt $3 a^{2 l o x / 1 l o x}$, TNAP-Cre] and [Dnmt $3 a^{\text {2lox/1lox }}$, Dnmt $3 b^{2 l o x / 1 l o x}$, TNAP-Cre] females were crossed with $\left[\mathrm{X}^{\mathrm{GFP}}, \mathrm{Y}\right]$ males, and embryos were recovered from the pregnant females at E8.5. In the embryo proper of female embryos derived from the mutant mothers, expression of the GFP transgene on the Xp was observed, just as in the embryo proper of control females, due to random X-inactivation (Fig. 3). By contrast, no GFP expression was ob-
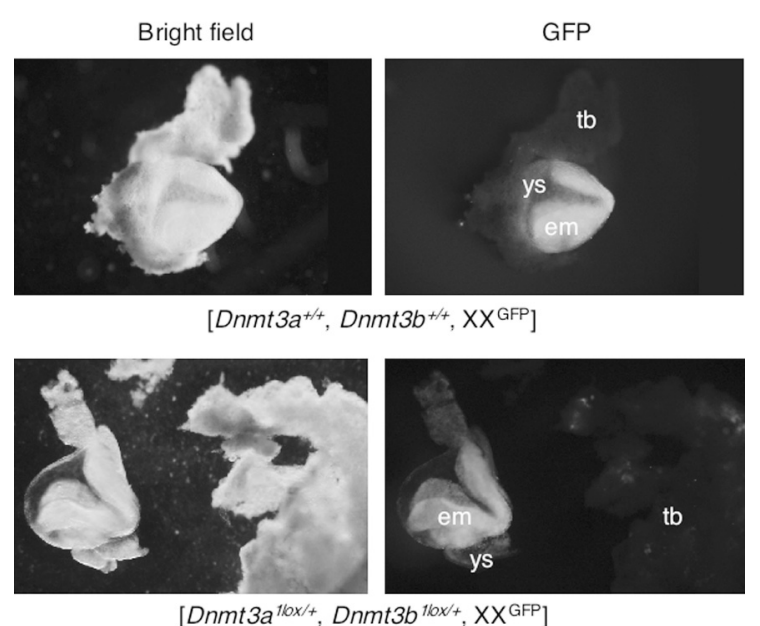

Figure 3. Lack of expression of a GFP transgene in the trophoblast of E8.5 embryos derived from [Dnmt3a 2lox/1lox, Dnmt3b 2 lox/llox , TNAP-Cre] females crossed with $\mathrm{X}^{\mathrm{GFP}} \mathrm{Y}$ males. Expression of GFP from the $\mathrm{Xp}$ is observed in the embryo proper (as a result of random X-inactivation) but not in the trophoblast (because of preferential Xp-inactivation) of both wildtype (top) and mutant (bottom) embryos ([Dnmt3allox/+ Dnmt $\left.\left.3 b^{\text {hlox/+ }}, \mathrm{XX}^{\mathrm{GFP}}\right]\right)$. This suggests, although indirectly, that the Xm derived from the conditional mutant females has the imprint to be active in the trophoblast. em, embryo proper; ys, yolk sac; tb, trophoblast.

served from the Xp in the trophoblast of female embryos derived from the mutant mothers as well as those from the control mothers (Fig. 3). These results exclude the possibility of random X-inactivation in the trophoblast and suggest, although indirectly, that a loss of the de novo DNA methyltransferases in the female germ line does not affect the imprinted $\mathrm{X}$-inactivation.

\section{CONCLUSIONS}

Based on the studies described here, we now begin to obtain a comprehensive view of the role of de novo DNA methyltransferases in the initiation of autosomal imprinting and X-inactivation. First, we found that Dnmt3a is required for the initiation of autosomal imprinting in both the paternal and maternal germ lines. Notably, we showed that a trophoblast-specific gene Mash2, which does not require Dnmtl for the maintenance of its imprinted state, does require Dnmt $3 a$ for the initiation of imprinting in the maternal germ line. Second, we found that the initiation of random $\mathrm{X}$-inactivation can occur normally in the absence of Dnmt3a and Dnmt3b. Third, we showed that a disruption of both Dnmt $3 a$ and Dnmt $3 b$ in the maternal germ line does not affect the imprinted X-inactivation in the trophoblast. This seems to indicate that the $\mathrm{Xm}$ derived from the mutant females has the imprint to remain active. The role of Dnmt3a and Dnmt $3 b$ in the imprinting of $\mathrm{Xp}$, however, remains an open question. These findings are summarized in Table 1, in terms of necessity for DNA methylation, together with the previous findings on the maintenance of these phenomena.

Autosomal imprinting and X-inactivation share a number of molecular features. These include cis-acting con- 
trol centers, long-distance regulation, association with noncoding and antisense RNAs, involvement of histone modifications and chromatin-associated factors, and differential DNA methylation (Lee 2003). These features lead to the proposal that $\mathrm{X}$-inactivation and autosomal imprinting may have a common origin (Lee 2003). Indeed, marsupials such as kangaroos show paternal-specific imprinted X-inactivation (Sharman 1971), just as the trophoblast of mice, and X-inactivation of this type is thought to be the early form of dosage compensation. Since de novo DNA methylation is required for the initiation of autosomal imprinting, it will be important to establish whether the same mechanism is involved in the initiation of imprinted X-inactivation. By contrast, we clearly showed that the initiation of random $\mathrm{X}$-inactivation, which may be the more recent form of dosage compensation, does not require de novo DNA methylation. This indicates that, even if $\mathrm{X}$-inactivation and autosomal imprinting have a common origin, a very different molecular mechanism evolved afterward to achieve random Xinactivation in the epiblast lineages.

\section{ACKNOWLEDGMENTS}

We thank A. Nagy and H. Lomeli (Samuel Lunenfeld Research Institute) for TNAP-Cre mice; M. Okabe (Osaka University) for $\mathrm{X}^{\mathrm{GFP}}$ mice; K. Shiota and $\mathrm{S}$. Tanaka (The University of Tokyo) for help in laser microdissection; $T$. Ohhata for photographing the $\mathrm{X}^{\mathrm{GFP}}$ embryos; and all members of our laboratories for help, discussion, and encouragement throughout this work. The work was supported in part by Grants-in-Aids from the Ministry of Education, Culture, Sports, Science, and Technology of Japan to H.S. and Grants from the National Institutes of Health to E.L.

\section{REFERENCES}

Beard C., Li E., and Jaenisch R. 1995. Loss of methylation activates Xist in somatic but not in embryonic cells. Genes Dev. 9: 2325.

Bour'chis D., Xu G.L., Lin C.S., Bollman B., and Bestor T.H. 2001. Dnmt3L and the establishment of maternal genomic imprints. Science 294: 2536.

Brockdorff N. 2002. X-chromosome inactivation: Closing in on proteins that bind Xist RNA. Trends Genet. 18: 352.

Caspery T., Cleary M.A., Baker C.C., Guan X.-J., and Tilghman S.M. 1998. Multiple mechanisms regulate imprinting of the mouse distal chromosome 7 gene cluster. Mol. Cell. Biol. 18: 3466.

Fitzpatrick G.V., Soloway P.D., and Higgins M.J. 2002. Regional loss of imprinting and growth deficiency in mice with a targeted deletion of KvDMR1. Nat. Genet. 32: 426.

Goto Y. and Takagi N. 1998. Tetraploid embryos rescue embryonic lethality caused by an additional maternally derived $\mathrm{X}$ chromosome in the mouse. Development 125: 3353.

Guillemot F., Nagy A., Auerbach A., Rossant J., and Joyner A.L. 1994. Essential role of Mash-2 in extraembryonic development. Nature 371: 333.

Guillemot F., Caspary T., Tilghman S.M., Copeland N.G., Gilbert D.J., Jenkins N.A., Anderson D.J., Joyner, A.L., Rossant J., and Nagy A. 1995. Genomic imprinting of Mash2, a mouse gene required for trophoblast development. Nat. Genet. 9: 235.

Hata K., Okano M., Lei H., and Li E. 2002. Dnmt3L cooperates with the Dnmt3 family of de novo DNA methyltransferases to establish maternal imprints in mice. Development 129: 1983.

Huynh K.D. and Lee J.T. 2003. Inheritance of a pre-inactivated paternal X chromosome in early mouse embryos. Nature 426: 857.

Kaneda M., Okano M., Hata K., Sado T., Tsujimoto N., Li E., and Sasaki H. 2004. Essential role for de novo DNA methyltransferase Dnmt3a in paternal and maternal imprinting. Nature 429: 900.

Lee J.T. 2003. Molecular links between X-inactivation and autosomal imprinting: $\mathrm{X}$-inactivation as a driving force for the evolution of imprinting? Curr. Biol. 13: R242.

Li E., Beard C., and Jaenisch R. 1993. Role for DNA methylation in genomic imprinting. Nature 366: 362.

Li E., Bestor T., and Jaenisch R. 1992. Targeted mutation of the DNA methyltransferase gene results in embryonic lethality. Cell 69: 915.

Lomeli H., Ramos-Mejia V., Gertsenstein M., Lobe C.G., and Nagy A. 2000. Targeted insertion of Cre recombinase into the TNAP gene: Excision in primordial germ cells. Genesis 26: 116.

Lyon M.F. 1961. Gene action in the X-chromosome of the mouse (Mus musculus L). Nature 190: 372.

Okamoto I., Tan S.-S., and Takagi N. 2000. X-chromosome inactivation in XX androgenetic mouse embryos surviving implantation. Development 127: 4137.

Okamoto I., Otte A.P., Allis C.D., Reinberg D., and Heard E. 2004. Epigenetic dynamics of imprinted X-inactivation during early mouse development. Science 303: 644

Okano M., Bell D.W., Haber D.A., and Li E. 1999. DNA methyltransferases Dnmt3a and Dnmt3b are essential for de novo methylation and mammalian development. Cell 99: 247.

Panning B. and Jaenisch R. 1996. DNA hypomethylation can activate Xist expression and silence X-linked genes. Genes Dev. 10: 1991.

Reik W. and Walter J. 2001. Genomic imprinting: Parental influence on the genome. Nature Rev. Genet. 2: 21.

Sado T., Okano M., Li E., and Sasaki H. 2004. De novo methylation is dispensable for the initiation and propagation of $\mathrm{X}$ chromosome inactivation. Development 131: 957.

Sado T., Fenner M.H., Tan S.-S., Tam P., Shioda T., and Li E. 2000. X-inactivation in the mouse embryo deficient for Dnmt1: Distinct effect of hypomethylation on imprinted and random X inactivation. Dev. Biol. 225: 294.

Sharman G.B. 1971. Late DNA replication in the paternally derived X chromosome of female kangaroos. Nature 230: 231.

Tada T., Obata Y., Tada M., Goto Y., Nakatsuji N., Tan S.-S., Kono T., and Takagi N. 2000. Imprint switching for non-random X-chromosome inactivation during mouse oocyte growth. Development 127: 3101.

Takagi N. and Sasaki M. 1975. Preferential inactivation of the paternally derived $\mathrm{X}$ chromosome in the extraembryonic membranes of the mouse. Nature 256: 640 .

Takagi N., Sugimoto M., Yamaguchi S., Ito M., Tan S.-S., and Okabe M. 2002. Nonrandom X chromosome inactivation in mouse embryos carrying Searle's T(X;16)16H translocation visualized using $\mathrm{X}$-linked $l a c Z$ and GFP transgenes. Cytogenet. Genome Res. 99: 52.

Tanaka M., Puchyr M., Gertsenstein M., Harpal K., Jaenisch R., Rossant J., and Nagy A. 1999. Parental-origin-specific expression of Mash2 is established at the time of implantation with its imprinting mechanism highly resistant to genome-wide demethylation. Mech. Dev. 87: 129.

Thornhill A.R. and Burgoyne P.S. 1993. A paternally imprinted $\mathrm{X}$ chromosome retards the development of the early mouse embryo. Development 118: 171. 


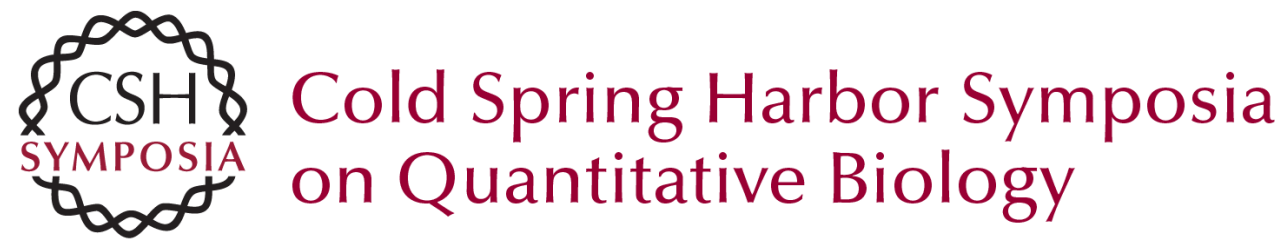

\section{Role of De Novo DNA Methyltransferases in Initiation of Genomic Imprinting and X-Chromosome Inactivation}

M. KANEDA, T. SADO, K. HATA, et al.

Cold Spring Harb Symp Quant Biol 2004 69: 125-130

Access the most recent version at doi:10.1101/sqb.2004.69.125

References This article cites 29 articles, 9 of which can be accessed free at: http://symposium.cshlp.org/content/69/125.full.html\#ref-list-1

\section{License}

Email Alerting

Receive free email alerts when new articles cite this article - sign up in Service the box at the top right corner of the article or click here. 